\title{
Evaluation of nucleic acid testing [NAT] of blood donors
}

\author{
Chaithanya $\mathrm{K}^{1, *}$, Shivakumar $\mathrm{S}^{2}$ \\ ${ }^{1}$ Assistant Professor, ${ }^{2}$ Professor, Dept. of Pathology, Mandya Institute of Medical Sciences, Mandya, Karnataka, India
}

Corresponding Author: Chaithanya K

Email: chaithanya.krishnappa@gmail.com

Received: $22^{\text {nd }}$ June, 2018

Accepted: $26^{\text {th }}$ June, 2018

\begin{abstract}
Context: Human immunodeficiency virus (HIV), hepatitis C virus (HCV), and hepatitis B virus (HBV) are easily transmitted through infected blood, to prevent this serological screening of blood units is a routine norm. Many cases could be missed if the testing is done in window period. To overcome this and to provide an additional protection many tests with better sensitivity and specificity like Nucleic acid testing (NAT) have been tried.

Aims: To observe NAT reactivity trend for transfusion transmittable diseases, and to correlate ELISA and NAT methods in detecting HIV, HBV and HCV.

Materials and Methods: A six years retrospective observational study conducted in Blood bank, Department of Pathology. ELISA and ID-NAT results of the donor samples for HIV, HBV and HCV were collected in a specially designed proforma and analyzed.

Statistical Analysis used: Data entered in Microsoft excel sheets, means and percentages were calculated.

Results: In 6years 52,417 units of blood collected, 44,600(85.08\%) were voluntary donors and 7,817(14.92\%) replacement donors. There were 521(0.99\%) cases of HBV, $98(0.19 \%)$ HIV and $48(0.09 \%)$ HCV and two cases of HIV-HBV co-infection. About 557 were positive by both ELISA and NAT method (1.06\%). Fifty eight cases were positive only by ID-NAT method and negative on serological testing accounting to the NAT yield of 1 in 893 cases. There were 2 cases of HIV-2 which were missed by NAT and picked up in ELISA.

Conclusions: Screening of blood and blood products using dual testing with high sensitivity serological assays and NAT helps to detect potentially infectious diseases in all phases of infection.
\end{abstract}

Keywords: ID-NAT, ELISA, Transfusion transmittable diseases.

\section{Introduction}

Blood and blood products transfusion plays a very essential role in health care system. Requirement of blood in India is about 12 million units per day. With the demand of this magnitude, safe and quality supply of blood and blood products becomes the concern. ${ }^{1}$ Blood is a potential source of transmitted diseases. Human immunodeficiency virus (HIV), hepatitis C virus $(\mathrm{HCV})$, and hepatitis $\mathrm{B}$ virus $(\mathrm{HBV})$ are easily transmitted through infected blood. Prevalence of these viruses among donors in India is about $0.24 \%$ for HIV, $0.43 \%$ for $\mathrm{HCV}$ and $1.18 \%$ for $\mathrm{HBV}$ which is very high compared to the developed and other developing countries. $^{2}$ To ensure safe blood supply WHO has called for $100 \%$ voluntary donation. In India paid donors have been banned since January 1998. However in our country family and replacement donors still provide more than $45 \%$ of required blood and number of replacement donors is more in hospital based blood banks where emergency requirement of blood and blood products are high. Studies have shown that transfusion transmittable infections (TTI's) are high in replacement donors compared to voluntary donors. ${ }^{3}$ Serological screening of blood donors for transmissible agents play a major role to decrease the risk of transfusion of infected units. Enzyme linked immune sorbent assays i.e. ELISA method is generally used for screening for the presence of antibodies however the time for the appearance of these antibodies may take few weeks known as 'window period'. The donor blood continues to be infective during this period. Many cases could be missed if ELISA is done in this period subjecting the recipient susceptible for infection. To overcome this and to provide an additional protection many tests with better sensitivity and specificity have been tried. Nucleic acid testing (NAT) is one among them which is based upon the technique of direct amplification and detection of viral nucleic acids rather than antibody production by the immune system of the infected person. NAT is thus able to detect viruses during the 'window period'. This allows for earlier detection of infection and further decreases the possibility of transmission via transfusion. ${ }^{4}$ The present study is planned to evaluate NAT in our blood bank and to compare its effectiveness against ELISA.

\section{Aims and Objectives}

1. To observe NAT reactivity trend for transfusion transmittable diseases among blood donors in our blood bank.

2. To correlate ELISA and NAT methods in detecting $\mathrm{HIV}, \mathrm{HBV}$ and HCV.

\section{Materials and Methods}

It is a six years retrospective record based observational study conducted in Blood bank, 
Department of Pathology. ELISA and NAT reports of HIV, HBV and HCV were collected in a specially designed proforma from the blood bank records. Both the tests are routinely done on the donor blood samples. Two pilot samples are collected from each donor bag in EDTA vaccutainers. One sample is centrifuged in REMI R-8C centrifuge instrument and the obtained plasma is used to run ELISA tests for HIV, HBsAg and HCV as per the Standard Operative Procedures prepared according to the NACO guidelines. HIV fourth generation kit and third generation kits of HBV and $\mathrm{HCV}$ are used [BIORAD serological test kits] in our blood bank. Reading is taken in the ROBONIK automated ELISA reader and washer. Results are tabulated. Repeat test is done on the positive cases. Other pilot sample is sent to NIMHANS Blood bank the same day of collection of blood where NAT is done on all the samples individually by Procleix Utrio Plus Elite Assay. Where in transcription mediated amplification (TMA) based screening for HIV-1 RNA, HCV RNA and HBV DNA takes place simultaneously in a single tube by transcription mediated amplification method. This happens in three steps Target capture, Target amplification by TMA and Detection of the amplification products with chemiluminescent probes by the hybridization protection assay. Results were sent through mail and for every positive cases the blood bag was quarantined and two repeat samples were sent on which the analysis was repeated and the results were confirmed and final results are generated. Thus obtained final data of both ELISA and Individual Donor Nucleic Acid Testing [ID-NAT] were collected in a specially designed proforma, tabulated and analysed. So obtained results data was collected in the proforma and analysed.

Inclusion Criteria: Based on the NACO guidelines all healthy donors donating blood in our blood bank and in the voluntary donation camps conducted by the blood bank.

Exclusion Criteria: Based on the NACO guidelines donors not eligible to donate blood were excluded from the study like-

1. Age group $<18$ and $>60$ years.

2. Known cases of HIV, HCV, HBV, syphilis and malaria positive patients.

3. Donors suffering from acute illness, malignancies, cardiac diseases.

4. Females who are pregnant, breast feeding, and during periods.

\section{Results}

In six year retrospective study from December 2011 to November 2017 total number of blood donations in our blood bank was 52,417. Among them 44,600 were voluntary donations accounting to $85.08 \%$ and remaining 7,817 were replacement donors accounting to $14.92 \%$. All blood samples were tested for HIV, HBV and HCV by both ELISA and ID-NAT methods. Among them $669(1.28 \%)$ cases were positive. There were $521(0.99 \%)$ cases positive for HBV, $98(0.19 \%)$ cases positive for HIV and 48 $(0.09 \%)$ cases for HCV and 2 cases of HIV and HBV co-infection. [Table 1]

Among 52,417 samples tested 557 cases were positive by both ELISA and NAT methods accounting to $1.06 \%$ of all donations in which $450(0.86 \%)$ cases were positive for HBV, $84(0.16 \%)$ cases for HIV and $23(0.04 \%)$ cases for HCV. [Table 2]

There were 58 cases which were positive only by ID-NAT method and negative on serological testing accounting for $0.11 \%$ of all donations. All serological testing was repeated on these samples which turned out to be negative and all repeat consecutive tests with two more samples from the representative blood bag was positive by ID-NAT. Out of 58 cases 49 were positive for HBV, 4 cases were positive for HCV, 3 cases for HIV and 2 cases were found out to be HIV and HBV co-infection. The combined NAT yield accounts to 1 in 893 cases. [Table 2]

There were 54 cases which were positive only by ELISA. There were 10 cases of seropositive for HIV. On repeat examination it was found out that two cases were positive for HIV-2 which cannot be picked by the NAT method since there was no programming to pick this strain. The other 8 cases on repeat serological testing found to be inconsistent. There were 21 cases of $\mathrm{HCV}$, repeat testing on these samples showed that only 6 cases were positive on two consecutive tests. Remaining 15 cases were positive only by one method of serology and negative by other ELISA methods and card tests. However repeat NAT test on all cases were negative. Out of 23 cases which were positive for HBV by ELISA, 10 were positive in first stage of analysis by ID-NAT but discriminatory assays and tests on repeat samples were non reactive. [Table 2]

Majority of the cases i.e. 382 were seen in the age group 26-40 years. Thirty five of 58 NAT yield cases belonged to this age group. Among 669 positive donors only one was female who was positive for HBV and remaining were males. [Table 3]

Table 1: Distribution of Positive cases

\begin{tabular}{|l|c|c|c|c|c|}
\hline Year & Total & ELISA \& NAT & NAT & ELISA & Total \\
\hline 2011 & 587 & 10 & - & 2 & 12 \\
\hline 2012 & 7710 & 101 & 7 & 23 & 131 \\
\hline 2013 & 7624 & 100 & 19 & 18 & 137 \\
\hline 2014 & 6963 & 80 & 9 & 6 & 95 \\
\hline
\end{tabular}




\begin{tabular}{|l|c|c|c|c|c|}
\hline 2015 & 9662 & 90 & 9 & 2 & 101 \\
\hline 2016 & 9698 & 85 & 7 & 3 & 95 \\
\hline 2017 & 10173 & 91 & 7 & & 98 \\
\hline Total & 52417 & 557 & 58 & 54 & 669 \\
\hline
\end{tabular}

ELISA- Enzyme Linked Immuno Sorbant Assay, NAT - Nucleic Acid Testing

Table 2: Year wise distribution of individual cases

\begin{tabular}{|c|c|c|c|c|c|c|c|c|c|c|}
\hline \multirow[b]{2}{*}{ Year } & \multicolumn{3}{|c|}{ ELISA \& NAT } & \multicolumn{3}{|c|}{ Only NAT } & \multicolumn{3}{|c|}{ Only ELISA } & \multirow[t]{2}{*}{ Total } \\
\hline & HIV & HBV & HCV & HIV & HBV & HCV & HIV & HBV & $\mathrm{HCV}$ & \\
\hline 2011 & 3 & 7 & - & - & - & - & - & 2 & - & 12 \\
\hline 2012 & 15 & 82 & 4 & 2 & 5 & & 15 & 4 & 4 & 131 \\
\hline 2013 & 17 & 82 & 1 & 1 & 15 & 3 & 4 & 1 & 13 & 137 \\
\hline 2014 & 8 & 65 & 7 & - & 9 & - & 1 & 3 & 2 & 95 \\
\hline 2015 & 10 & 77 & 3 & - & 8 & 1 & 2 & - & - & 101 \\
\hline 2016 & 16 & 63 & 6 & 1 & 6 & - & 1 & - & 2 & 95 \\
\hline 2017 & 15 & 74 & 2 & - & 7 & - & - & - & - & 98 \\
\hline Total & 84 & 450 & 23 & 4 & 50 & 4 & 23 & 10 & 21 & 669 \\
\hline
\end{tabular}

HIV- Human Immunodeficiency Virus, HBV- Hepatitis B Virus, HCV- Hepatitis C Virus

Table 3: Age wise distribution of positive cases

\begin{tabular}{|l|c|c|c|c|c|}
\hline \multicolumn{1}{|c|}{ Test } & Number & $\mathbf{1 8 - 2 5}$ & $\mathbf{2 6 - 4 0}$ & $\mathbf{4 1 - 5 9}$ & Total \\
\hline \multirow{4}{*}{ ELISA \& NAT } & HIV & 28 & 55 & 1 & 84 \\
\cline { 2 - 6 } & HBV & 172 & 246 & 32 & 450 \\
\cline { 2 - 6 } & HCV & 8 & 15 & - & 23 \\
\hline \multirow{3}{*}{ Only NAT } & HIV & 1 & 2 & 1 & 4 \\
\cline { 2 - 6 } & HBV & 10 & 30 & 10 & 50 \\
\cline { 2 - 6 } & HCV & - & 3 & 1 & 4 \\
\hline \multirow{3}{*}{ Only ELISA } & HIV & 6 & 4 & - & 10 \\
\cline { 2 - 6 } & HBV & 6 & 15 & 2 & 23 \\
\cline { 2 - 6 } & HCV & 7 & 12 & 2 & 21 \\
\hline Total & & 238 & 382 & 49 & 669 \\
\hline
\end{tabular}

HIV- Human Immunodeficiency Virus, HBV- Hepatitis B Virus, HCV- Hepatitis C Virus

\section{Discussion}

In India under Sec 3(b) of drug and cosmetics act human blood is considered as drug and is regulated by the rules of the act. The inspection and licensing of blood banks in India is covered under this act of the Ministry of Health and Family Welfare. The rules of this act stipulate that before transfusion, mandatory testing should be done of each sample of donor blood for hepatitis $\mathrm{B}(\mathrm{HBV})$, HIV, hepatitis C virus (HCV), syphilis and malaria. ${ }^{5}$

Screening of donated blood for HIV-1 and -2 and hepatitis B was started in India in 1989 and for antibodies to $\mathrm{HCV}$ in 1995 by serological testing by ELISA. ${ }^{6}$ However if the donor is in window period of the infection or is an occult carrier then he can still be seronegative and have a potential risk for transmission of TTIs and thus serological testing cannot assure complete safety against TTIs. ${ }^{7}$

To overcome this residual risk and to provide an additional layer of safety, evaluation of donor blood by NAT was started in early 1990's in developed countries and was introduced in developing countries in late 1990's. According to Ministry of Health and Family Welfare data [last updated on 2015] in India, there are
2760 licensed blood banks which includes both government and private institutions. According to the study by Ghosh K and Mishra K in 2017 around 58 blood banks have implemented NAT test to screen the donor blood which accounts to $2.1 \%$ of the blood banks. $^{5}$

NAT detects viral ribonucleic acid (RNA) or deoxyribonucleic acid (DNA) by the amplification method. Early in the course of an infection, NAT detects low levels of viral genetic material present in the blood. NAT is thus able to detect viruses during the 'window period' allowing earlier detection of infection and further decreasing the possibility of transmission via blood transfusion. NAT also detects mutants and occult cases. ${ }^{8,9}$

Individual donor-NAT [ID-NAT] and Minipool NAT [MP-NAT] are currently used in conjunction with serological tests in many countries in Asia, Australia, Europe and North America and Asia. ${ }^{10}$ In ID-NAT detection of HIV-1, HBV and HCV takes place simultaneously in a single tube and unlike MP-NAT single donor specimen is screened at a time rather on pooled sample thus increasing the specificity of the test and decreasing the time take to give results. However 
since the test is done on individual samples separately cost of these test is high. The window period for detection by ID-NAT by ultrio plus is 4.7 days for HIV1, 2.2 days for HCV and 16.3 days for HBV. ${ }^{11,12}$ The corresponding window periods for serological test are 15-20 days, 2-26 weeks and 50-150 days respectively. ${ }^{13}$

Voluntary to replacement donors ratio in our study is $85.08 \%: 14.98 \%$ which can be compared to the study done by Jain et $\mathrm{al}^{16}$ in which the ratio was $84.65 \%$ : $15.35 \%$, however in few other studies replacement donors were more than voluntary donors $35.2 \%: 64.8 \%,,^{13} \quad 3,225: 8999^{14}$ and $99.3 \%: 0.7 \%{ }^{11}$. Reason for more number of voluntary donors in our study is that in our institution according to NACO guidelines family donors are also considered under voluntary donor's category.

The prevalence of HBV, HIV and HCV among donors in our study was $0.99 \%, 0.19 \%$ and $0.09 \%$ respectively. HIV prevalence is close to the NACO figures i.e. $0.2 \%$, however, $\mathrm{HBV}$ and $\mathrm{HCV}$ prevalence rate is relatively less which are $1.4 \%$ and $0.4 \%$ respectively. ${ }^{6}$ Similar less prevalence was observed in a study by Naidu et al with prevalence rates being HIV $0.08 \%$, HCV $0.09 \%$, and HBV $0.70 \% .{ }^{15}$ However in a study by Jain et al prevalence rate of HCV was $0.63 \%$, HBsAg $1.5 \%$ and HIV $0.33 \% .{ }^{16}$ Following was the observation by Makroo et al in their study HIV $0.24 \%$, Hep B $1.18 \%$ and Hep C $0.43 \% .^{2}$ In a multicentric evaluation study done by Makroo et al the prevalence rate observed was HBV $1.12 \%$, HCV $0.33 \%$ and HIV $0.26 \% .{ }^{14}$ In a study done by Kumar et al the prevalence rate was $0.12 \%$ for $\mathrm{HIV}, 0.99 \%$ for $\mathrm{HCV}$ and $1.95 \%$ for HBsAg. ${ }^{13}$ Chandra $\mathrm{T}$ et al observed $0.11 \%$ of HIV, $0.16 \% \mathrm{HCV}$ and $1.67 \%$ for HBV. ${ }^{17}$ The difference in the prevalence of the TTI's in various studies are may be because of the regional variation of the incidence of the same.

In our study majority of the donors found to be seropositive belong to the age group 26-40 years 382 cases, followed by 18-25years 238 cases and 41-59 years 49cases. In a study by Jain et al similar distribution was observed. ${ }^{16}$ In a study by Chatterjee et al they observed majority of NAT cases i.e 5 were seen in the age group 31-45years. ${ }^{9}$ More number of reactive donors were observed in all studies in third decade is mainly because number of donors younger than 25 years are relatively less. This lack of enthusiasm is mainly because lack of awareness and motivation in young population.

Among all the positive cases only one was female donor. Similar male to female differences were observed in the studies by Jain et al where in $97.71 \%$ males: $2.29 \%$ females $42.7: 1{ }^{16}$ in the multicentric study by Makroo et al percentage of reactive male donors was twice more than reactive female donors (1.85\%:0.85\%). ${ }^{14}$ More cases of positivity is observed in males among most of the studies including our study is mainly because in our country female donors are very less. Thus the same has been reflected in reactive donors.

The NAT yield in our study is 1 in 893.2. That is out of every 893 non seroreactive cases one person turned out to be positive by NAT method. There were 58 NAT positive cases in our study that means 174 recipients would have been affected if these cases were not detected by NAT. Discriminatory assays showed individual NAT yield for HBV is 1 in 1,036 and for HIV and HCV are 1 in 12951. NAT yield in other studies across India varied from 1 in few hundreds to many thousands. In a study by Pathak et al there were 3 positive cases of 6587 negative samples and all were positive for $\mathrm{HBV}^{18}$ in a study by Jain et al there were 8 NAT positive cases with the NAT yield 1 in 2972 and again all cases were positive for HBV. ${ }^{16}$ In a study by Agarwal et al NAT yield was 1 in 610 with 1 HIV, 37 $\mathrm{HCV}, 73 \mathrm{HBV}$ and $10 \mathrm{HBV}-\mathrm{HCV}$ co-infections. ${ }^{19}$ In a multicentric study by Makroo et al they analyzed data from 8 centres and they observed a NAT yield of 1 in $1528(0.065 \%)$ with $6 \mathrm{HBV}$ cases, $1 \mathrm{HCV}$ case and 1 HIV-1 cases. In their study they also observed that NAT yield was more in hospital based blood banks. ${ }^{14}$

In another multicentric study by ghosh et al they observed that the NAT yield varied from 1:476 to 1:4403 in various studies and according to their pooled data showed HIV in 1:66,000, Hep C virus 1:5484 and Hep $\mathrm{B}$ in 1:1761 seronegative donors. ${ }^{20}$ In another study by Kumar et al combined NAT yield among blood donors was 1 in 753with 1 in HIV-1, $13 \mathrm{HCV}$ and $27 \mathrm{HBV}$ cases. ${ }^{13}$

The NAT yield rate from other blood banks in India is 1 in $3182,{ }^{21} 1$ in $2622^{9}$ In a study by Chandra T et al there were $158(0.44 \%)$ NAT only positive cases with $2 \mathrm{HIV}-1,2$ cases of HIV-2, 46 cases of HCV and 108 cases of HBV. ${ }^{17}$ In a study by Sultan et al in Pakistan NAT yield was 1 in 1143 with 1 in $1600 \mathrm{HBV}$ and 1 in $4,000 \mathrm{HCV}^{11}$ Salim $\mathrm{H}$ et al in Saudi Arabia in their study observed 7 positive cases $(5 \mathrm{HCV}$ and 2 HIV-1) in 12,032 seronegative cases. ${ }^{22}$ NAT yield in other countries in various studies are as follows in US ( 1 in 2 million for HIV and HCV), ${ }^{23}$ Germany ( 1 in 431843), ${ }^{24}$ Japan (1 in 48262), ${ }^{25}$ Singapore ( 1 in $24567),{ }^{21}$ and Thailand $\left(1\right.$ in 25000). ${ }^{21}$ in western Europe 1 in 6 million. ${ }^{26}$ Variation in the number of NAT yield could be mainly because of (i) different prevalence rate of that particular disease in that particular region. (ii) NAT yield was relatively less in few studies mentioned above in few institutions where in they used $4^{\text {th }}$ generation ELISA tests the sensitivity and specificity of which are pretty good. (iii) NAT yield was less where as seropositivity was high in certain studies where in they observed that viral load was comparatively very less in the samples which was failed to be picked up by NAT.

Prevalence of TTI's among donors is considered as surrogate marker of infections in the general population. ${ }^{27}$ In majority of the studies discussed above 
including our study it was observed that NAT yield was very high for Hep B suggesting high number of occult infection cases and cases in window period in the general population thus suggesting stringent measures has to be taken to screen actual number of Hep B cases in the society, to assess the immunization status and about considering public policy measures to immunize all for Hep B as the donors alone doesn't mirror the exact prevalence since donors below 18 , above 60 , with other co-morbid conditions are excluded from screening and also female donors are very less.

In countries like India being highly populated and with high incidence rates of TTI's, significant number of donors in window periods can be picked up by NAT. However it cannot alone be used as a screening test, as at times viral load may be of undetectable levels by NAT but antibody response may still be present. And cases of HIV-2 can be missed. Thus highly sensitive serological assays are also required for the safety of the blood for transfusion.

\section{Conclusion}

Thus to conclude in order to provide safe blood transfusion stringent measures in donor screening including promoting voluntary blood donation, screening of blood and blood products using dual testing with high sensitivity serological assays and NAT, which helps to detect potentially infectious diseases in all phases of infection is recommended to enhance the safety of blood and blood products transfusion.

\section{References}

1. NACO | National AIDS Control Organisation. Available rom :http://www.naco.gov.in/NACO/ National_AIDS_Control_Program/Services_for_Preventi on/ Access_to_Safe_blood/. [Last cited on 2018 Feb 06].

2 Makroo RN, Hegde V, Chowdhry M, Bhatia A, Rosamma NL. Seroprevalence of infectious markers and their trends in blood donors in a hospital based blood bank in North India. Indian J Med Res. 2015;142:317-22.

3 Voluntary blood donation programme -An operational Guideline, 2007. Available from:

http://www.nacoonline.org/upload/Policies \& Guidelines/29, voluntary blood donation.pdf

4 Hollinger FB, Liang TJ. Hepatitis B virus. In: Knipe DM et al. eds. Field virology, $4^{\text {th }}$ ed. Philadelphia, Lippincot Williams and Wilkins, 2001:2971-3036

$5 \quad$ Blood bank. Central Drugs Standard Control Organization. Guidelines for blood banks (updated 2012 Jul 26). Available from: www.cdsco.nic.in/forms/list, accessed on Jan 10, 2018.

6 Bhatia R Blood transfusion services in developing countries of South-East Asia. Transfus Today. 2005;65:5647-53.

7 Hans R, Marwaha N. Nucleic acid testing - benefits and constraints. Asian J Transfus Sci. 2014;8(1):2-3.

8 Coste J. Implementation of donor screening for infectious agents transmitted by blood by nucleic acid technology: Update to 200. Vox Sang. 2005;88:289-303.

9 Chatterjee $\mathrm{K}$ et al. Individual donor nucleic acid testing for blood safety against HIV-1 and Hepatitis B and C viruses in a tertiary care hospital. The National Medical Journal of India Vol 25, No.4,2012

10 Engelfriet CP, Reesink HW, Hernandez JM, Sauleda S, O'Riordan J, Pratt D, et al. Implementation of donors screening for infectious agents transmitted by blood by nuclic acid technology. Vox Sang. 2002;82:87-111.

11 Sultan S. Multiplex real-time RT-PCR assay for transfusion transmitted viruses in sero - negative allogenic blood donors: an experience from Southern Pakistan. Malaysian J Pathol. 2017;39(2):149-154

12 Taurifi HA. Evaluation of nucleic acid testing for blood donors: One year study. Int J Blood Transfus Immunohematol. 2015;5:19-25.

13 Kumar R, Gupta S, Kaur R, Gupta M. Individual donor nucleic acid testing for human immunodeficiency virus 1 , hepatitis $\mathrm{C}$ virus and hepatitis B virus and its role in blood safety. Asian Journal of Transfusion Medicine. 2015;9(2):199-202.

14 Makroo R N. Multicenter evaluation of individual donor nucleic acid testing (NAT) for simultaneous detection of human deficiency virus $-1 \& 2$ hepatitis B \&C viruses in Indian blood donors. Indian J Med Res. 127, February 2008, 140-147.

15 Naidu NK, Barucha ZS, Sonawane V, Ahmed I. Nucleic Acid Testing: Is it the only answer for safe blood in India?. Asian Journal of Transfusion Medicine. 2016;10(1):79-83.

16 Jain R, Aggarwal P, Gupta G N. Need for Nucleic Acid Testing in countries with high prevalence of Transfusion - Transmitted Infections. ISRN Hematology. 2012, Article ID 718671, 5 pages doi:10.5402/2012/718671

17 Hans R, Marwaha N. Nucleic acid testing - benefits and constraints. Asian J Transfus Sci. 2014;8(1):2-3.

18 Pathak S, Chandrashekar M. Transfusion transmittable infections - Seroprevalence among blood donors in a tertiary care hospital of Delhi. Asian journal of transfusion medicine. 2013;7(2):116-118.

19 Agarwal N, Chatterjee K, Coshic P, Borgohain M. Nucleic acid testing for blood banks: An experience from a tertiary care centre in New Delhi, India. Transfusion and apheresis science. 2013;49(3):482-484.

20 Ghosh K, Mishra K. Nucleic acid amplification testing in Indian blood banks: A review with perspectives. Indian journal of Pathology and Microbiology. 2017;60(3):313318.

21 Makroo RN. Impact of routine individual blood donor nucleic acid testing (ID-NAT) for HIV-1, HCV and HBV on blood safety in a tertiary care hospital. Apollo Med. 2007;4:9-12.

22 Selim, H.M., ElBashaar, M.A. \& ElWakil, S.G. Donor minipool NAT screening for HBV, HCV, and HIV: a 2year experience in a private hospital in Saudi Arabia. Comp Clin Pathol. 2014;23:1125.

23 Stramer SL, Glynn SA, Kleinman SH, Strong DM, Caglioti S, Wright DJ, et al. Detection of HIV-1 and $\mathrm{HCV}$ infections among antibody-negative blood donors by nucleic acidamplification testing. $N$ Engl J Med. 2004:351:760-8.

24 Hourfar MK, Jork C, Schottstedt V, Weber-Schehl M, Brixner V, Busch MP, et al. Experience of German Red Cross blood donor services with nucleic acid testing: Results of screening more than 30 million blood donations for human immunodeficiency virus-1, hepatitis $\mathrm{C}$ virus, and hepatitis $\mathrm{B}$ virus. Transfusion. 2008;48:1558-66.

25 Ohnuma $\mathrm{H}$. The first large- scale nucleic acid amplification testing (NAT) of donated blood using Multiplex reagent for simultaneous detection of $\mathrm{HBV}$, 
HCV and HIV-1 and significance of NAT for HBV. Microbiol Immunol. 2001;45(9): 667-672.

26 B. Singh. "Nucleic Acid Testing (NAT) screening of blood donors in India," A Project Report, International Hospital Federation Reference Book, 2008/2009.

27 M. Meena, T. Jindal, and A. Hazarika, "Prevalence of hepatitis $\mathrm{B}$ virus and hepatitis $\mathrm{C}$ virus among blood donors at a tertiary care hospital in India: a five-year study,” Transfusion. 2011;51(1):198-202.

How to cite this article: Chaithanya $\mathrm{K}$, Shivakumar S. Evaluation of nucleic acid testing [NAT] of blood donors. Indian J Pathol Oncol. 2018;5(4):536-541. 